\title{
LWOS: A Localization Method Without On-Body Sensor in Wireless Sensor Networks
}

\author{
Shuangquan Wang ${ }^{1}$, Ningjiang Chen ${ }^{2}$, Xin Chen ${ }^{2}$, Jie Yang ${ }^{1}$, and Jun Lu ${ }^{1}$ \\ ${ }^{1}$ Institute of Image Processing \& Pattern Recognition, Shanghai Jiaotong University, \\ Shanghai, China, 200240 \\ \{wangshuangquan, jieyang, lujun0328\} asjtu.edu.cn \\ ${ }^{2}$ Philips Research East Asia, Shanghai, China, 200070 \\ \{james.nj.chen, mylan.chen\}@philips.com
}

\begin{abstract}
In many applications of wireless sensor networks (WSNs), the location information of users is very important. In this paper we present a localization method without on-body sensor (LWOS). The basic idea is that when a person is standing between a pair of transceivers, the human body will attenuate the received signal. From the detected attenuation of Received Signal Strength Indication (RSSI), LWOS can detect and localize people directly utilizing the wireless communication in WSNs. No additional sensor is needed and users do not need to wear a sensor node any more. A signal-shielding device is used at the transmitter side to minify the interference of RSSI variability from multi-path effects. Experiment results show a good capability of localizing a single user in an indoor environment.
\end{abstract}

\section{Introduction}

The advances in wireless sensor networks (WSNs) foster a growing interest in location-aware applications. The key distinguishing feature of such an application is that it provides services to users based on their physical location information [1]. This information can also be used to deduce what people are doing with the assumption that a person's activity is correlated to his/her location.

There are several available localization techniques [2], such as Global Positioning System (GPS), ultrasound, Infra-Red (IR), and radio based methods. GPS is proven to be effective, but too expensive to be widely adopted by cheap and small devices [3]. Ultrasound and IR based methods are usually used in indoor environments, while they require additional hardware to be added to off-the-shelf sensor nodes. Comparatively, radio based method is the most attractive localization technology because radio transceiver is already available in each node and less power consumption is needed.

Radio based localization methods can be classified into two classes: range-based and range-free [3]. The difference between them is whether absolute point-to-point distance or angle estimate is used. The former includes the methods based on Time of Arrival (TOA) [4], Time Difference of Arrival (TDOA) [5, 6], Angle of Arrival (AOA) [7] and Received Signal Strength Indication (RSSI) [1]. The latter includes Centroid [8], DV-Hop [9] and Amorphous [10]. 
However almost all above methods require people to wear a wireless sensor node in order to calculate the related information, such as distance, with other reference sensor nodes. Although in this way, the system may provide accurate location information, our experience indicates that users often feel uneasy and uncomfortable when wearing sensors. In this paper we present a localization method without onbody sensor (LWOS) based on RSSI. It utilizes the normal wireless communication to detect and localize people. Compared with above localization methods, LWOS does not force users to wear sensor nodes.

The rest of the paper is organized as follows. In Section 2, we introduce the previous work in RSSI-based localization methods. Section 3 describes the rationale of LWOS method. Experiment results will be illustrated in Section 4 and some LWOS associated topics will be discussed in Section 5. Finally, we conclude the paper in Section 6.

\section{Related Works}

In RSSI techniques, theoretical and empirical models are proposed to translate signal strength into distance estimates. RADAR [1] proposes a RF based system for locating and tracking users inside buildings. It records and processes signal strength information at multiple base stations, which are purposely positioned to provide overlapping coverage in the area of interest. It combines empirical measurements with signal propagation model to determine user location. SpotON [11] creates a SpotON object tag as a distance estimator based on radio signal strength analysis and focuses mostly on the hardware and embedded systems aspects. The method in [2] analyzes the experimental data and uses a specific signal attenuation model to provide distance information from the raw RSSI data. Ecolocation [12] determines the location of unknown nodes by examining the ordered sequence of RSSI measurements taken at multiple reference nodes. In [13], RSSI values are used to estimate the one-hop distance in a grid.

However the carefully designed set of indoor and outdoor experiments demonstrate that many factors, such as radio calibration, antenna orientation, background noise and obstacles, may cause RSSI to be unpredictable. The layout of the rooms in the building, the placement of base stations, and the location of the mobile user all have an effect on the received signal. In [14], the author indicates that all these RSSI based localization algorithms have a high error rate.

\section{LWOS}

The key of high error rate existing in RSSI based localization methods is the RSSI variability, especially in indoor environments. The RSSI variability means that the signal becomes unpredictable due to reflection, diffraction, scattering, and refraction. We propose to use a signal-shielding device, which is a metal can with one head open, 
for each transmitter to weaken the interference of RSSI variability. After using the signal-shielding device, LWOS becomes realizable.

\subsection{RSSI Variability}

The RSSI in a radio communication system is affected by many factors, which can be divided into two categories: propagation media related and device related $[14,15,16$, 17]. Media properties include the media type, the background noise and some other environmental factors, such as the temperature and obstacles within the propagation media. Device-related factors include antenna type (directional or omni-directional), transmission power, antenna gains (at both the transmitter and receiver) and receiver sensitivity etc.

In order to decrease the interference of these factors, we set some conditions in our study of RSSI variability. The transmitters and receivers have the same type of monopole antennas. All sensor nodes are pre-tested to have similar RSSI values when there is no person between them. The antenna directions of a pair of transmitter and receiver are parallel and opposite. Fully charged batteries are used for all sensor nodes to ensure that they work well. Additionally there is little background noise in the same radio frequency band and some other factors, such as temperature and humidity, have almost no effect on the RSSI variability as tested.

These conditions relieve the interference of many factors. When a person keeps still or leaves the room for a while, the signal will become stable gradually. However the RSSI value still varies greatly when the person walks around a pair of transceivers several meters away in a room. It is hard to judge from the change in RSSI value whether the person is present or not. According to [14], the main factor is the multipath fading and shadowing in the RF channel. In indoor environments, the electromagnetic signal may be reflected when it encounters the walls, furniture and other objects. Since the radio travels at the speed of light, it is too fast for the receiver to distinguish the original signal and the reflected signal. It can only measure the received signal strength for both. For this reason, the presence and movement of the person interfere the radio propagation.

\subsection{Weaken the RSSI Variability by a Signal-Shielding Device}

Since the antenna has a spherical radio propagation model in theory, a reasonable solution is to install a signal-shielding device at each transmitter to decrease the multipath effect. A metal can with one head open is used in our experiments. Fig.1 shows the sensor deployment in the metal can and the sketch map of signal propagation with the shielding device.

The RSSI value after using the shielding device is shown in Fig.2. The packet ID is the index of packets sent from the transmitter to the receiver and reflects the time. The protuberant parts of rectangle line and diamond line represent two cases respectively: 1) a person standing on the line-of-sight between one pair of sensor nodes; and 2) going across the line-of-sight between another pair. The RSSI value varies within $1 \mathrm{dBm}$ to $4 \mathrm{dBm}$ when no person is present, which is much smaller than the one without the signal-shielding device. 


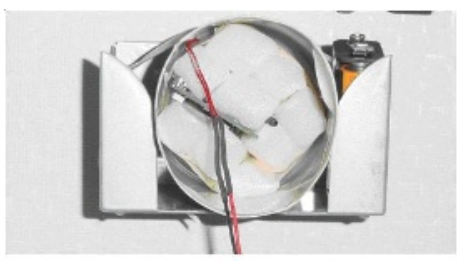

(a)

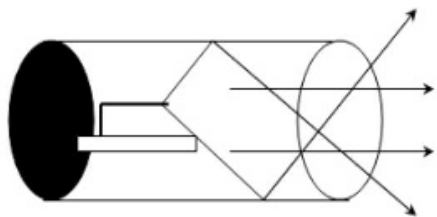

(b)

Fig. 1. (a) A metal can as a signal-shielding device with a sensor node inside; (b) Theoretic signal propagation diagram after using the signal-shielding device



Fig. 2. The RSSI value when the signal is focused

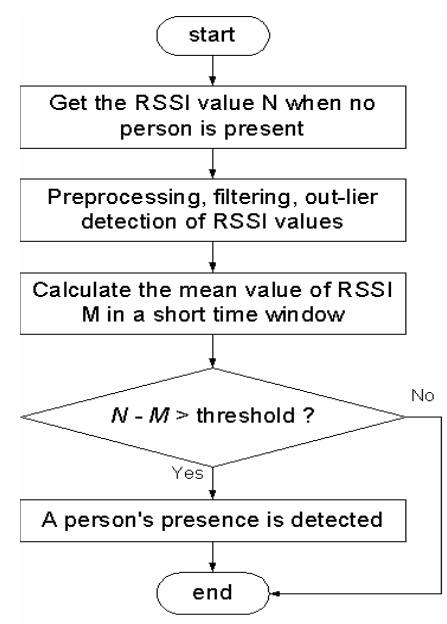

Fig. 3. One cycle of presence detection

\subsection{Localization}

LWOS locates a person by detecting the attenuation of RSSI when the human body absorbs some of the signal energy or reflects the signal to other directions. The body interference is related to the frequency of the radio signal. Normally, the higher the frequency is, the greater the attenuation is. The $2.4 \mathrm{GHz} \mathrm{RF}$ is used in our experiments. In fact, the $2.4 \mathrm{GHz}$ is the frequency of water resonance used by a microwave oven.

In our experiments, the RSSI value for each pair of transceivers is supposed to be $\mathrm{N} d B m$ when there is no person and $\mathrm{M} d B m$ when a person is standing in the detection strip, which is a narrow strip area on the line-of-sight between them. The person can be detected by comparing the change in RSSI value with a threshold, which is an average estimation of the RSSI change when the person is present. Different size and orientation of human body can result in different amounts of signal attenuation. The detection result is sensitive to the threshold, which needs to be selected carefully. One cycle of presence detection is described in Fig. 3. 
Based on the presence detection, LWOS can localize and track the movement of a person. Several detection strips are used to cover the detection area as shown in Fig. 4. If a person stands at any position in this area, at least one detection strip will be covered. When the detection area is too large for the detection strips to cover, LWOS can localize the person in one of the four triangular sub-areas based on the order of covered strips (coming in or going out the sub-area). Or several detection strips are linked in a close loop, then the person can be localized in the detection area of the close loop as shown in Fig. 5.



Fig. 4. An detection area covered by six detection strips (lines linking two nodes)

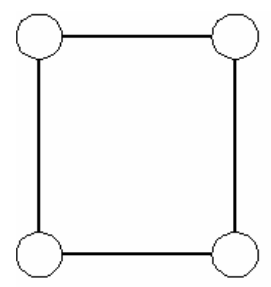

Fig. 5. An detection area covered by four detection strips in a close loop

\section{Experiments and Results}

Many experiments are done to measure the RSSI threshold for finding the detection strip of each pair of transceivers and evaluate the LWOS method.

\subsection{Experiment Setup}

A meeting room in our laboratory is used as the experimental environment. There is a table in the center of the room with several chairs around. Sensor nodes are attached to the wall, legs of tables or put along the windowsill.

The sensor node uses the Chipcon CC2420 IEEE 802.15.4 radio transceiver [14] in the physical layer. The transmission power is programmable in eight steps from $-24 \mathrm{dBm}$ to $0 \mathrm{dBm}$ [18]. A built-in received signal strength indicator gives an 8-bit digital value as RSSI value. The sensor node uses a monopole antenna, which is $2.9 \mathrm{~cm}$ long as the optimal antenna length according to the CC2420's datasheet [19].

In our system, when the receiver received a message from the transmitter, the transmission power $P$, their IDs and the RSSI value are packed and forwarded to a base station, which is connected to a PC for processing as in Section 3.3.

\subsection{RSSI Threshold for Finding the Detection Strip}

In LWOS, the main parameters are transmission power, the distance and offset angle between the transmitter and the receiver. To get the RSSI threshold for finding the 
detection strip between a pair of transceivers, we fix one transmitter (Tx) and three receivers $(\mathrm{Rx})$ in an indoor environment as shown in Fig.6 (a).

The transmission power is $-5 \mathrm{dBm}$ and all sensor nodes are put at $50 \mathrm{~cm}$ from the ground. The whole area is divided into grids. The RSSI matrix of Rx 2 is shown in Fig.6 (b). The x varies form $-90 \mathrm{~cm}$ to $90 \mathrm{~cm}$ and y varies from $0 \mathrm{~cm}$ to $150 \mathrm{~cm}$, with an interval of $15 \mathrm{~cm}$ respectively. The person stands at each cross point of the grids between two nodes and faces to the Y direction.



(a)

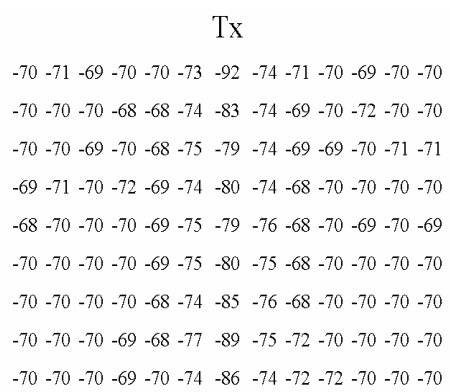

(b)

Fig. 6. (a) The coordinates of one transmitter and three receivers; (b) The RSSI matrix of the receiver 2 with the presence of a person

When there is no person, the RSSI value at Rx 2 is $-70 \mathrm{dBm}$. Fig.7 (a) shows the RSSI difference matrix, which is the result of the RSSI matrix subtracting the original value for no person. The area with a big RSSI difference is a detection strip on the line-of-sight between two nodes. Its width is about $20 \mathrm{~cm}$. With the distance from the line-of-sight increases, the difference decreases sharply.

Many experiments indicate that setting the RSSI threshold to $8 \mathrm{dBm}$ is reasonable. If the threshold is too big, there are some blind spots, where the system cannot detect the human presence, and it is difficult to find a detection strip. Contrarily if the threshold is too small, the detection strip is too wide and the detection is sensitive to the RSSI variability. With a threshold of $8 \mathrm{dBm}$, the binary detection matrix is shown in Fig.7 (b), where 0 indicates no person and 1 indicates detected presence. The results of $\operatorname{Rx} 1$ and $\mathrm{Rx} 3$ are got with the same experimental condition as $\mathrm{Rx} 2$.

\subsection{One Transmitter to Multiple Receivers}

The experiment result also shows that one transmitter can be paired with several receivers, which can be placed at any place within the valid detecting distance and offset angle. All detection strips are long, narrow and have little interference with each other. The detection strip of the middle pair is the most regular one. With the 
increase of offset angle, the detection strip becomes irregular and nonlinear. Our experiment result indicates that the offset angle is better to be no more than $\pm 45^{\circ}$.

\subsection{Detecting Distance}

The detecting distance for each transmission power level is measured. The pair of transceivers is deployed face to face as the middle pair as shown in Fig.6 (a). Within the detecting distance, the RSSI value at any position in the detection strip should be larger than $-85 \mathrm{dBm}$. Otherwise the RSSI value is less than $-93 \mathrm{dBm}$ when a person is present and most messages may be lost because the signal is too weak. On the other hand even though the RSSI value is high enough, the interference of a person to the signal is trivial when the distance is too large. We measure the detecting distance that satisfies the two demands above for eight transmission power levels. The results are shown in Table 1.

Table 1. The detecting distance for different transmission power level

\begin{tabular}{ccccccccc}
\hline Power Level $(\mathrm{dBm})$ & -25 & -15 & -10 & -7 & -5 & -3 & -1 & 0 \\
\hline Distance $(\mathrm{cm})$ & 50 & 150 & 180 & 200 & 230 & 250 & 270 & 280 \\
\hline
\end{tabular}

Except the lowest transmission power level, the detecting distances are all equal or larger than $150 \mathrm{~cm}$ and the largest one is $280 \mathrm{~cm}$. With the increase of offset angle, the detecting distance decreases accordingly. Fig. 8 shows the relation between the offset angle and the valid detecting distance when the transmission power is $-5 \mathrm{dBm}$. The offset angle is within $\pm 45^{\circ}$. Otherwise, the detection strip becomes irregular and there are too many blind spots on the line-of-sight.

\subsection{Presence Detection and Localization}

Experiment results show that the presence detection using our method is encouraging. Usually when a certain transmission power level is chosen, the distance between a pair of transceivers is fixed and the furniture arrangement is supposed to have no great change, the experiment result is repeatable.

Some localization and tracking experiments are done in the environment as shown in Fig.9. Totally five detection areas (A E) are defined in Fig. 9, each of which is covered by several detection strips as indicated by the dot lines. The arrow lines indicate the trace of the person. In most cases LWOS can localize and track the person very well.

Because LWOS cannot distinguish the people between two transceivers and there is no person identification information available, it is often used to localize a single user. Multiple users can be localized only when they do not appear in the same area simultaneously. As shown in Fig. 4 and Fig. 5, LWOS can only locate the person in certain detection areas. The localization in higher accuracy can be achieved by adding more pairs of transceivers in an area. Each pair will divide the detection area into smaller ones. 


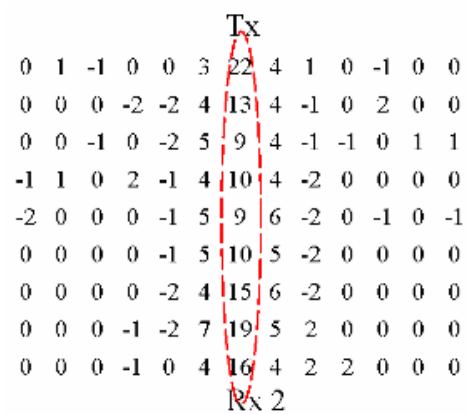

(a)

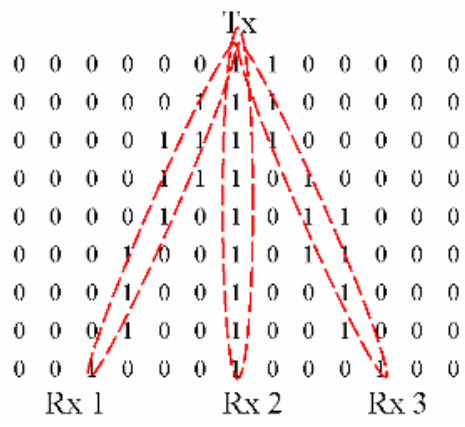

(b)

Fig. 7. (a) The RSSI difference matrix of receiver 2; (b) The binary detection matrix of three pairs of transceivers (shared one transmitter)

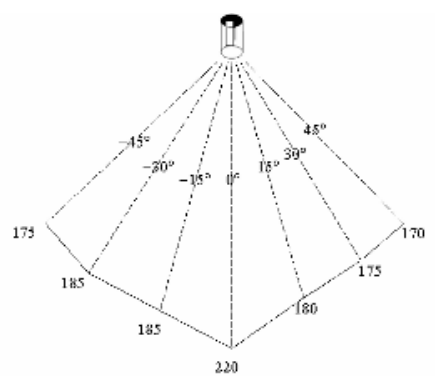

Fig. 8. The relation between the offset angle and the distance at $-5 \mathrm{dBm}$ transmission power

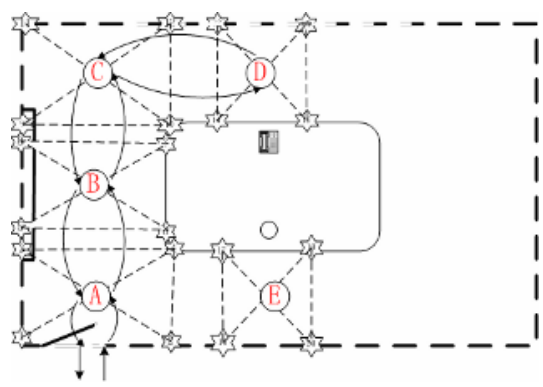

Fig. 9. The performance of LWOS in localization and tracking

\section{Discussions}

Some experiments are done to examine the varieties of signal propagation model in the front area and back area of the transmitter respectively. The front area is covered by the beam radio signal, while the back area is not covered. The difference of RSSI values between with and without the signal-shielding device when no person is present in indoor environments is shown in Fig.10. The experiment setting in Fig. 10 is the same as that in Fig. 7.

Fig. 10 shows that the RSSI differences in both front area and back area are irregular. Obviously the multi-path still exists. Fig. 10 (b) indicates that the signalshielding device weakens the signal in the back area. This decreases the indirect signal reflected from the wall, furniture and some other objects. The experiment results in outdoor environments are similar with those in indoor environments. The result indicates that the shielding device, compared with a directional antenna, is not the best choice. 
It is impossible to formulate the relation of distance, transmission power, offset angle and some other parameters. Using a threshold to judge the human presence is the best available method at present. It is better to use a mechanism to adjust the threshold dynamically.

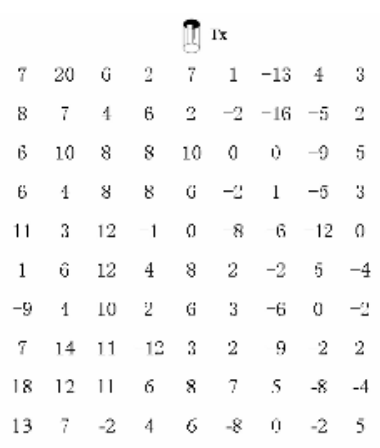

(a)



(b)

Fig. 10. (a) The difference of RSSI value distribution in the front area between with and without a signal-shielding device; (b) The difference of RSSI value distribution in the back area between with and without a signal-shielding device. Both are in the indoor environment.

\section{Conclusions}

In this paper we present LWOS, a localization system without on-body sensor in indoor environments. LWOS detects the presence of a person based on the RSSI (Radio Signal Strength Indication) attenuation when the radio penetrates the human body. A signal-shielding device is used at each transmitter to decrease the interference of RSSI variability. Each pair of transceivers provides a detection strip. By using several pairs of transceivers, a place can be covered by those detection strips. Or several detection strips are linked in a close loop to localize the person in the closed area.

LWOS makes the user feel comfortable without the need of wearing a sensor node. It also directly utilizes the wireless communication in WSNs to provide the localization service without any additional sensor. Although LWOS presents a good capability in presence detection, localization and tracking, it currently can only localize a single person. Multiple people are can be localized only when they are not in the same area simultaneously.

Experiment results show that the multi-path is not mainly originating from the reflection of wall, furniture and objects, but from the reflection of the inner side of the shielding device. We find the RSSI value is still irregular in the detection strip between the transmitter and the receiver. Our following research includes: 1) stabilizing the signal attenuation from the human body by using a directional antenna instead of a signal-shielding device; 2) setting the RSSI threshold in a dynamic way. 


\section{References}

1. P. Bahl and V. N. padmanabhan. RADAR: An In-Buliding RF-based User Location and Tracking System. Proceedings of the IEEE Infocom 2000, Tel-Aviv, Israel, March 2000.

2. http://groups.csail.mit.edu/drl/journal_club/papers/nana.dankwa.ee.pdf

3. T. He, C. Huang, B. M. Blum, J. A. Stankovic, and T. Abdelzaher. Range-Free Localization Schemes in Large-Scale Sensor Networks. In Proc. of the Intl. Conference on Mobile Computing and Networking (MOBICOM), September 2003.

4. L. Girod and D. Estrin. Robust Range Estimation Using Acoustic and Multimodal Sensing. In: Proc IEEE/RSJ Int'l Conf Intelligent Robots and System (IROS'01), Vol.3, Maui, Hawaii, USA. 2001.

5. N. Priyantha, A. Chakraborthy and H. Balakrishnan. The Cricket Location-Support System. In: Proc Int'l Conf on Mobile Computing and Networking, August 6-11, 2000, Boston, MA.

6. A. Savvides, C. C. Han and B. M. Srivastava. Dynamic Finge-Grained Localization in AdHoc Networks of Sensors. In: Proc 7th Annual Int'l Conf on Mobile Computing and Networking (MobiCom). Rome, Italy. July 2001.

7. D. Niculescu and B. Nath. Ad Hoc Positioning System (APS) using AoA, INFOCOM'03, San Francisco, CA, 2003.

8. N. Bulusu, J. Heidemann and D. Estrin. GPS-less Low Cost Outdoor Localization for Very Small Devices. IEEE Personal Communications Magazine, 7(5): 28-34, October 2000.

9. D. Niculescu and B. Nath, DV Based Positioning in Ad-Hoc Networks. In Journal of Telecommunication Systems, 2003.

10. R. Nagpal. Organizing a Global Coordinate System from Local Information on an Amorphous Computer. A. I. Memo 1666, MIT A. I. Laboratory, August 1999.

11. J. Hightower, G. Boriello and R. Want. SpotON: An Indoor 3D Location Sensing Technology Based on RF Signal Strength. University of Washington CSE Report, February 2000.

12. K. Yedavalli, B. Krishnamachari, S. Ravula, and B. Srinivasan. Ecolocation: A Technique of RF Based Localization in Wireless Sensor Networks. In Proceedings of Information Processing in Sensor Networks (IPSN), Los Angels, CA, April 2005.

13. R. Stoleru and J. Stankovic. Probability Grid: A Location Estimation Scheme for Wireless Sensor Network. In Proceedings of Sensor and Ad Hoc Communications and Networks Conference (SECON), Santa Clara California, October 4-7, 2004.

14. D. Lymberopoulos, Q. Lindsey and A. Savvides. An Empirical Analysis of Radio Signal Strength Variability in IEEE 802.15.4 Networks using Monopole Antennas. ENALAB Technical Report 050501, 2005.

15. N. Reijers, G. Halkes and K. Langendoen. Link Layer Measurements in Sensor Networks. In: Proc. of the First IEEE International Conference on Mobile Ad hoc and Sensor Systems, MASS 2004, 24-27 October, Fort Lauderdale, FL, 2004.

16. G. Zhou, T. He, S. Krishnamurthy and J. A. Stankovic. Impact of Radio Irregularity on Wireless Sensor Networks. In Proceedings of ACM SenSys, November 2004.

17. G. Gaertner and V. Cahill. Understanding Link Quality in 802.11 Mobile Ad Hoc Networks. In IEEE Internet Computing, January February 2004.

18. D. Lymberopoulos and A. Savvides. Xyz: A Motion-Enabled, Power Aware Sensor Node Platform for Distributed Sensor Network Applications. In IPSN, SPOTS track, April 2005.

19. http://www.chipcon.com 